in vivo $34: 3109-3114(2020)$

doi:10.21873/invivo.12144

Review

\title{
Lipid-lowering Drugs and Neurocognitive Function: A Systematic Review
}

\author{
GEORGIOS KYRIAKOS ${ }^{1}$, LOURDES VICTORIA QUILES-SÁNCHEZ ${ }^{2}$, \\ EVANGELOS DIAMANTIS ${ }^{3}$, PARASKEVI FARMAKI ${ }^{4}$, NIKOLAOS GARMPIS ${ }^{5,6}$, \\ CHRISTOS DAMASKOS $^{6,7}$, SPYRIDON SAVVANIS ${ }^{8}$, ALEXANDROS PATSOURAS ${ }^{9}$, ATHANASIA STELIANIDI ${ }^{4}$, \\ ERRIKA VOUTYRITSA ${ }^{6}$, VASILIKI E. GEORGAKOPOULOU ${ }^{10,11}$ and ANNA GARMPI ${ }^{12}$
}

\author{
${ }^{1}$ Seccion de Endocrinologia y Nutrition, Hospital General Universitario Santa Lucia, Cartagena, Spain; \\ ${ }^{2}$ Centro de Salud Jesús Marín, Murcia, Spain; \\ ${ }^{3}$ Department of Endocrinology and Diabetes Center, G. Gennimatas General Hospital, Athens, Greece; \\ ${ }^{4}$ First Department of Pediatrics, Agia Sofia Children's Hospital, Medical School, \\ National and Kapodistrian University of Athens, Athens, Greece; \\ ${ }^{5}$ Second Department of Propedeutic Surgery, Laiko General Hospital, Medical School, \\ National and Kapodistrian University of Athens, Athens, Greece; \\ ${ }^{6}$ N.S. Christeas Laboratory of Experimental Surgery and Surgical Research, Medical School, \\ National and Kapodistrian University of Athens, Athens, Greece; \\ ${ }^{7}$ Renal Transplantation Unit, Laiko General Hospital, Athens, Greece; \\ ${ }^{8}$ Internal Medicine Department, Elpis General Hospital, Athens, Greece; \\ ${ }^{9}$ Second Department of Internal Medicine, Tzanio General Hospital, Piraeus, Greece; \\ ${ }^{10}$ Department of Pulmonology, Laiko General Hospital, Athens, Greece; \\ ${ }^{11}$ First Department of Pulmonology, Sismanogleio Hospital, Athens, Greece; \\ ${ }^{12}$ First Department of Propedeutic Internal Medicine, Laiko General Hospital, \\ Medical School, National and Kapodistrian University of Athens, Athens, Greece
}

\begin{abstract}
Background/Aim: Lipid-lowering drugs have been suggested to affect neurocognitive function. This review aimed to give the latest evidence on the way these agents affect neurocognitive function based on clinical trials. Materials and Methods: A systematic search concerning original studies from 2015 to 2020 was performed through the databases PubMed, EMBASE and Cochrane, according to the PRISMA (Preferred Reporting Items for Systematic reviews and Meta-Analyses) guidelines. The trials enrolled
\end{abstract}

This article is freely accessible online.

Correspondence to: Dr. Christos Damaskos, MD, MSc, Ph.D., NS Christeas Laboratory of Experimental Surgery and Surgical Research, Renal Transplantation Unit, Laiko General Hospital, Medical School, National and Kapodistrian University of Athens, 17 Agiou Thoma Street, Athens, 11527, Greece. Tel: +30 6948467790,e-mail: x_damaskos@yahoo.gr

Key Words: Lipid-lowering drugs, neurocognitive function, coronary heart disease, hypercholesterolemia, clinical trials, review. numerous patients and were conducted in different areas of the world. The terms used are cholesterol, lipid-lowering drugs, statins and cognitive function. Results: Eleven randomized trials met the inclusion criteria. The trials included patients suffering from cardiovascular conditions. In particular, patients with coronary heart disease, coronary heart disease risk equivalents and hypercholesterolemia were tested. The trials included evolocumab, alirocumab, statin, ezetimibe or placebo. Conclusion: Lipid-lowering drugs seem to have no significant effect on neurocognitive function, but further research specifically focused on this matter is needed.

Previous evidence has shown that the more risk factors for vascular disease one has in middle age, the greater the risk for dementia later in life. High risk cholesterol levels are among the risk factors. Evidence suggests that LDL is associated with cognitive impairment while HDL improves memory (1).

Cognitive impairment relates to attention, processing speed, learning, memory and verbal flexibility. Studies of two rare diseases, Down's syndrome and Niemann Pick type 
C, have found that cholesterol interferes with the process of cellular proliferation and this results in defective offspring of cells throughout the body, leading to various diseases, such as heart diseases and Alzheimer's disease $(2,3)$. Also, it has been found that people with high cholesterol have significantly more plaques in their brains and that high cholesterol is related to a reduced risk of cognitive impairment to the elderly (4). Furthermore, it has been observed that statins are associated with some protection against cognitive impairment, but as individuals grow, protection declines (4). Lately, proprotein convertase subtilisin-kexin type 9 (PCSK9) inhibitors have constituted a noteworthy class of medical treatment that significantly lower low-density lipoprotein cholesterol (LDL-C) via upregulation of LDL receptor availability (5). However, there is great concern whether these treatments affect neurocognitive function. This article reviews the latest evidence from randomized trials on this matter.

\section{Materials and Methods}

Three known databases (EMBASE, PubMed and Cochrane library) were systematically explored for retrieval of potentially relevant articles published from 2015 through 2020. The keywords used were cholesterol, lipid-lowering drugs, statins and cognitive function. Articles were also searched from references of the original papers and review articles. Articles concerning clinical trials were included. All duplicates were removed, and the remained records were screened for eligibility criteria. Studies suspected for bias were excluded. The research was performed in agreement with the PRISMA (Preferred Reporting Items for Systematic reviews and Meta-Analyses) guidelines as presented in Figure 1. The database resulted in 11 trials that met the inclusion criteria. They are all randomized trials that compare evolocumab and alirocumab either to each other or each to placebo or a statin. The number of patients in the presented trials ranged from 250 to 27,500 and was from different sites in many countries of the world (Table I).

\section{Results}

A recently developed and effective method for lowering LDL-C levels and reducing cardiovascular diseases is the employment of human mAbs inhibiting PCSK9. Two mAbs for LDL-C reduction, evolocumab and alirocumab, have been permitted by both the FDA and European Medicines Agency and both have affirmed a notable ability in reducing LDL-C along with a promising safety profile (6). Additionally, according to the Further Cardiovascular Outcomes Research that reported the PCSK9 inhibition in patients with elevated risk trial (7), patients presenting high cardiovascular risk were investigated at first. Evolocumab has been proven to decrease cardiovascular episodes. However, patients treated with either alirocumab or evolocumab provided significantly decreased levels of ontreatment plasma cholesterol in the majority of cases (mean level $30 \mathrm{mg} / \mathrm{dl}$ :FOURIER trial and even $<25 \mathrm{mg} / \mathrm{dl}$ : ODYSSEY LONG-TERM trial) (8), which is worrying in terms of potential unfortunate effects of these drugs, like neurocognitive disorders.

Some studies have observed similar neurocognitive adverse effects in patients receiving PCSK9 inhibitors and in control groups. In ODYSSEY LONG-TERM trial, medication based on alirocumab was related to a no notable increase in neurocognitive disorders such as memory impairment, amnesia and confusional state in patients being prone to present cardiovascular events. Similarly, in the reported studies ODYSSEY FH I, FH II and ODYSSEY HIGH FH and COMBO I (9-11), a small number of neurocognitive events was reported in patients treated with alirocumab [2 $(0.6 \%)$ in FH I, 1 in HIGH FH and no events were reported in FH II and COMBO I] or placebo [2 (1.2\%) in FH I, 1 (1.2\%) in FH II, COMBO I and HIGH FH]. On the other hand, the OSLER trial cited a slight increase of neurocognitive unfavorable events in patients that received evolocumab. When 11.1 months of treatment were completed, the overall incidence of declared neurocognitive events was detected and was found to be extremely low $(<1 \%)$. This outcome was not linked to the therapy of plasma LDL-C level (12). According to ODYSSEY OPTIONS I and II studies, as well as the ODYSSEY COMBO II trial (1315), alirocumab can be compared to ezetimibe, as adjuvant therapy to maximally tolerated statin therapy in patients at increased cardiovascular risk with insufficient controlled hypercholesterolemia. Not many neurocognitive episodes appeared in either group in both trials [4 $(0.8 \%)$ in patients that received alirocumab and $3(1.2 \%)$ in ezetimibe group in ODYSSEY OPTIONS II, 1 patient in each group in ODYSSEY COMBO II trial and none in ODYSSEY OPTIONS I]. Evolocumab vs. placebo treatment was studied in RUTHERFORD-2 study and no neurocognitive events were reported (16).

The Evaluating PCSK9 Binding Antibody Influence on Cognitive Health in High Cardiovascular Risk Subjects (EBBINGHAUS), constitutes a randomized and doubleblind study that utilized placebo-controlled tests, and was conducted to investigate the impact on neurocognitive testing, during the treatment, in patients treated with evolocumab combined with statin $v s$. statin alone. In order for cognitive tests to take place, the Cambridge Neuropsychological Test Automated Battery, a tablet-based tool assessing executive function, working memory, memory function, and psychomotor speed at first, weeks 24 and 48, every 48 weeks thereafter, and at the end, was utilized. The main endpoint was spatial working memory strategy index of principal function (SWMSI). The researchers aimed to investigate whether evolocumab would result in similar outcomes to placebo in the mean change in SWMSI from the beginning onwards. Investigators estimated that up to 


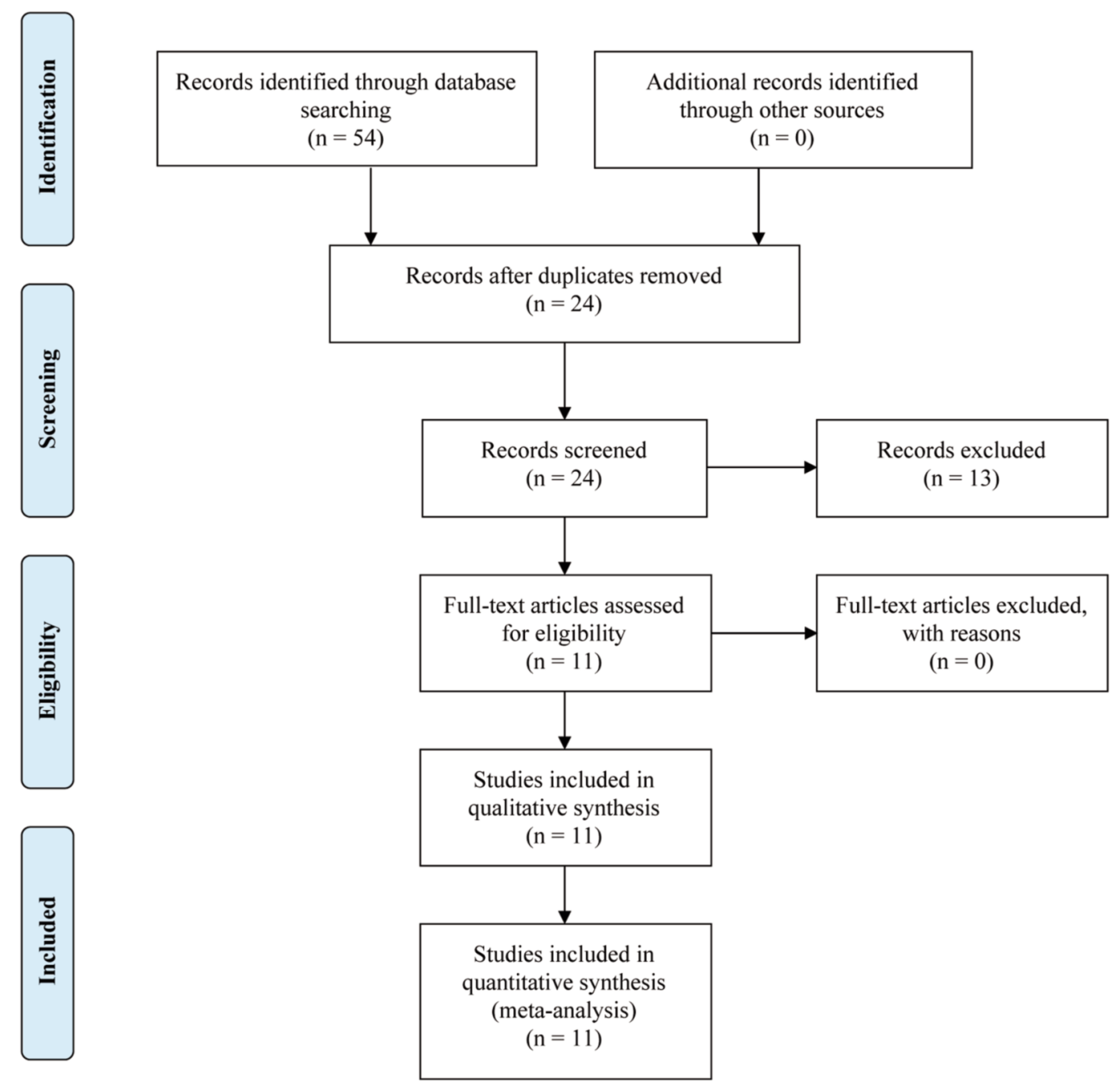

Figure 1. PRISMA flow diagram for the current literature review.

fifteen hundred participants without cognitive issues were required in order to have $97 \%$ power to identify noninferiority; they also set the noninferiority boundary at $20 \%$ of the standard deviation. Spatial Working Memory strategy index findings were similar between patients treated with evolocumab and placebo at baseline (17.8 for each) or at the end of the trial (17.5 vs. 17.6, respectively; $p<0.0001$ for noninferiority). Concerning the secondary endpoints, including additional neuropsychological items of Cambridge Neuropsychological Test Automated Battery, also provided similar results between patients randomized to medical treatment of evolocumab or placebo. Moreover, reports from patients and investigators regarding cognitive adverse events did not differ not only for placebo, but also for evolocumab. The analysis findings indicated that cognitive function was not influenced by on-treatment LDL-C status; therefore, cognitive performance in patients with an LDL-C $<25 \mathrm{mg} / \mathrm{dl}$ was alike to that in patients presenting higher plasma LDLC levels (17).

In 2017, Guigliano et al. conducted a study in which 15,281 patients were included. Patients received simvastatin $40 \mathrm{mg}$ plus ezetimibe $10 \mathrm{mg}$ or placebo for a median period of 6 years. As far as the neurocognitive dysfunction is concerned, neither longterm nor short-term adverse events appeared during treatment, even in patients with LDL-C levels lower than $30 \mathrm{mg} / \mathrm{dl}$ (18).

\section{Discussion}

Cholesterol and especially LDL cholesterol homeostasis has a pivotal role in the appropriate cellular function since it is a part of the cell membrane (19). The safety of new lipidlowering therapies and the combinations of these agents have attracted the interest of the research community. However, the long-term effects of the use of lipid-lowering drugs on 


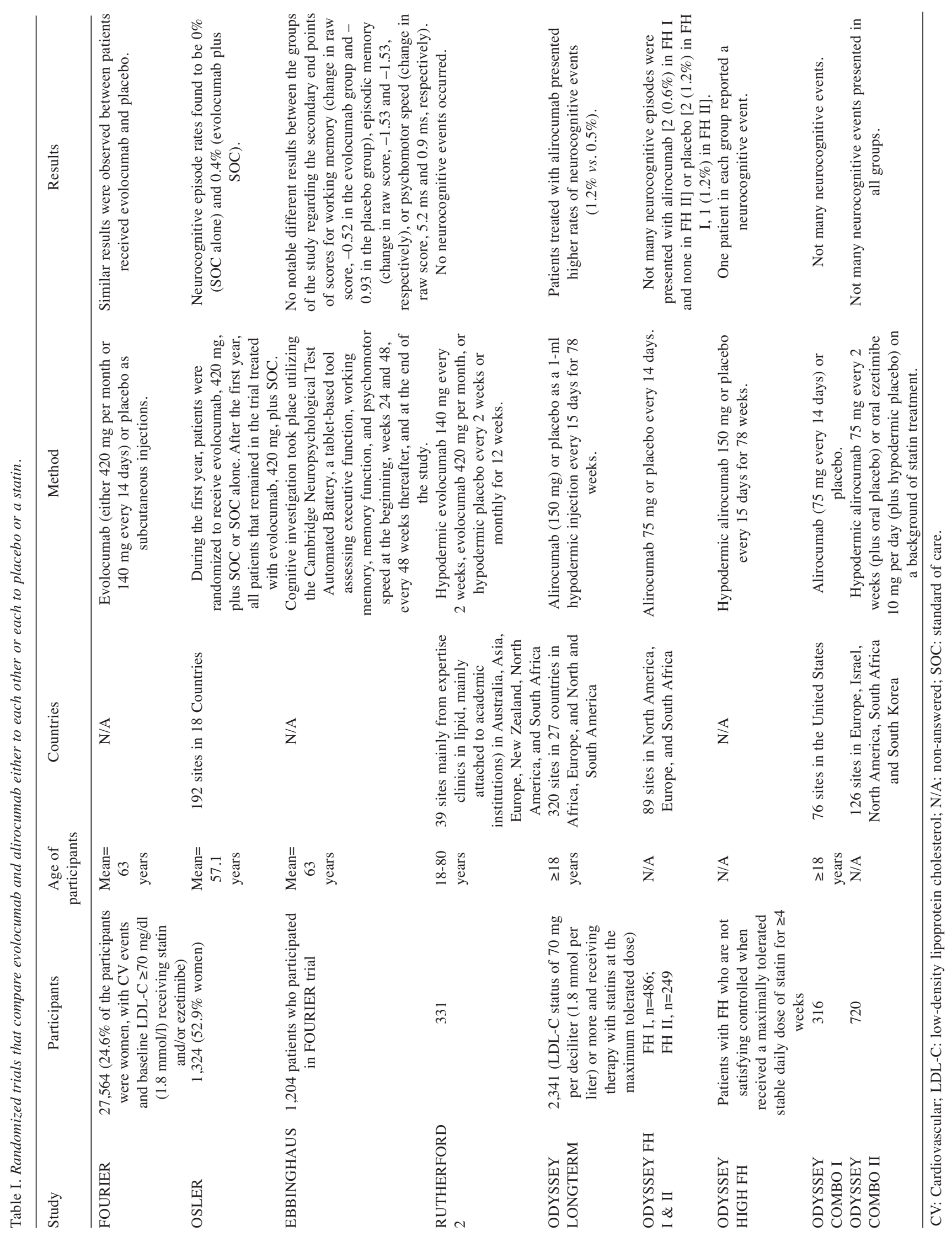


neurocognitive functioning still need to be investigated, as the increase in reported adverse outcome rates of 0.1 to $1.0 \%$ indicate that they are not uncommon (20).

Pooled data analysis on alirocumab showed that both patients treated with alirocumab and patients receiving placebo experienced comparable neurocognitive events (1.0\% and $0.9 \%)$ and the findings of neurocognitive episodes did not differ between patients that obtained a dose of LDL-C less or more than $25 \mathrm{mg} / \mathrm{dl}$ (21). That was also the case in a pooled data analysis on evolocumab, which presented rare neurocognitive unfavorable events that were compared in the different study groups $(0.1 \%$ in evolocumab vs. $0.3 \%$ in control groups) (22).

On the other hand, a more recent meta-analysis found a notable raise in neurocognitive adverse episodes among patients treated with PCSK9 inhibitor in comparison to patients who received placebo (23). However, since it included results from the Open-Label Study of Long-Term Evaluation against LDL Cholesterol trial and results obtained from its long-term extension, the same patients were tested twice in the same study. Once those findings were omitted, there was no correlation between PCSK9 therapy and neurocognitive adverse events (24). Nonetheless, in recent trials, the neurocognitive adverse events were reported by patients and no objective assessments of cognitive ability took place. Moreover, in most trials, no data on patients' beginning cognitive level were reported.

Our review, based on the latest studies, does not show any significant repercussion of neurocognitive events in patients taking lipid-lowering drugs. However, there are some limitations that affect our conclusions, such as the missing patients' cognitive status at the beginning of some trials, and the variety regarding the magnitude of LDL lowering, which may affect neurocognition.

\section{Conclusion}

In conclusion, neurocognitive dysfunction is a medical challenge, which is frequently met in people receiving drugs against dyslipidemia. Even though lipid-lowering drugs seem to be safe in terms of neurocognitive function, more trials specifically focusing on that issue need to be performed in order to have solid conclusions on the matter.

\section{Conflicts of Interest}

All the Authors declare that there are no conflicts of interest regarding this study.

\section{Authors' Contributions}

GK, ED and AG designed the study. GK, LVQS and ED wrote the article. PV, NG, CD, AP, EV and AG collected the data. GK, ED, SS and AG offered scientific advice. CD, NG, AS, AP and VEG critically revised the manuscript. AG was the supervisor.

\section{References}

1 Schultz BG, Patten DK and Berlau DJ: The role of statins in both cognitive impairment and protection against dementia: A tale of two mechanisms. Transl Neurodegener 7(7): 5, 2018. PMID: 29507718. DOI: 10.1186/s40035-018-0110-3

2 Zigman WB, Schupf N, Jenkins EC, Urk TK, Tycko B and Silverman W: Cholesterol level, statin use and Alzheimer's disease in adults with Down syndrome. Neurosci Lett 416(3): 279-284, 2007. PMID: 17353095. DOI: 10.1016/j.neulet. 2007.02 .023

3 Wang LJ and Song BL: Niemann-Pick C1-Like 1 and cholesterol uptake. Biochim Biophys Acta 1821(7): 964-972, 2012. PMID: 22480541. DOI: 10.1016/j.bbalip.2012.03.004

4 Svensson T, Sawada N, Mimura M, Nozaki S, Shikimoto R and Tsugane S: The association between midlife serum high-density lipoprotein and mild cognitive impairment and dementia after 19 years of follow-up. Transl Psychiatry 9(1): 26, 2019. PMID: 30659169. DOI: 10.1038/s41398-018-0336-y.

5 Banach M, Rizzo M, Nikolic D, Howard G, Howard V and Mikhailidis D: Intensive LDL-cholesterol lowering therapy and neurocognitive function. Pharmacol Ther 170: 181-191, 2017. PMID: 27865998. DOI: 10.1016/j.pharmthera.2016.11.001

6 Karatasakis A, Danek BA, Karacsonyi J, Rangan BV, Roesle MK, Knickelbine T, Miedema MD, Khalili H, Ahmad Z, Abdullah S, Banerjee S and Brilakis ES: Effect of PCSK9 inhibitors on clinical outcomes in patients with hypercholesterolemia: A meta-analysis of 35 randomized controlled trials. J Am Heart Assoc 6(12): e006910, 2017. PMID: 29223954. DOI: 10.1161/JAHA.117.006910

7 Katsiki N, Athyros VG, Mikhailidis DP and Mantzoros C: Proprotein convertase subtilisin-kexin type 9 (PCSK9) inhibitors: Shaping the future after the further cardiovascular outcomes research with PCSK9 inhibition in subjects with elevated risk (FOURIER) trial. Metabolism 74(9): 43-46, 2017. PMID: 28477848. DOI: 10.1016/j.metabol.2017.04.007

8 Robinson JG, Farnier M, Krempf M, Bergeron J, Luc G, Averna M, Stroes ES, Langslet G, Raal FJ, Shahawy MEI, Koren MJ, Lepor NE, Lorenzato C, Pordy R, Chaudhari U and Kastelein JJP: Efficacy and safety of alirocumab in reducing lipids and cardiovascular events. N Engl J Med 372(16): 1489-1499, 2015. PMID: 25773378. DOI: 10.1056/NEJMoa1501031

9 Kastelein JJ, Ginsberg HN, Langslet G, Hovingh GK, Ceska R, Dufour R, Blom D, Civeira F, Krempf M, Lorenzato C, Zhao J, Pordy R, Baccara-Dinet MT, Gipe DA, Geiger MJ and Farnier M: ODYSSEY FH I and FH II: 78 week results with alirocumab treatment in 735 patients with heterozygous familial hypercholesterolaemia. Eur Heart J 36(43): 2996-3003, 2015. PMID: 26330422. DOI: 10.1093/eurheartj/ehv370

10 Ginsberg HN, Rader DJ, Raal FJ, Guyton JR, Baccara-Dinet MT, Lorenzato C, Pordy R and Stroes E: Efficacy and safety of alirocumab in patients with heterozygous familial hypercholesterolemia and LDL-C of $160 \mathrm{mg} / \mathrm{dl}$ or higher. Cardiovasc Drugs Ther 30(5): 473-483, 2016. PMID: 27618825. DOI: $10.1007 / \mathrm{s} 10557-016-6685-\mathrm{y}$

11 Kereiakes DJ, Robinson JG, Cannon CP, Lorenzato C, Pordy R, Chaudhari $U$ and Colhoun HM: Efficacy and safety of the proprotein convertase subtilisin/kexin type 9 inhibitor alirocumab among high cardiovascular risk patients on maximally tolerated statin therapy: The ODYSSEY COMBO I 
study. Am Heart J 169(6): 906-915, 2015. PMID: 26027630. DOI: 10.1016/j.ahj.2015.03.004

12 Koren MJ, Sabatine MS, Giugliano RP, Langslet G, Wiviott SD, Kassahun H, Ruzza A, Ma Y, Somaratne R and Raal FJ: Longterm low-density lipoprotein cholesterol-lowering efficacy, persistence, and safety of evolocumab in treatment of hypercholesterolemia: Results up to 4 years from the open-label OSLER-1 extension study. JAMA Cardiol 2(6): 598-607, 2017. PMID: 28291870. DOI: 10.1001/jamacardio.2017.0747

13 Bays H, Gaudet D, Weiss R, Lima Ruiz J, Watts GF, GouniBerthold I, Robinson J, Zhao J, Hanotin C and Donahue S: Alirocumab as add-on to atorvastatin versus other lipid treatment strategies: ODYSSEY OPTIONS I randomized trial. J Clin Endocrinol Metab 100(8): 3140-3148, 2015. PMID: 26030325. DOI: $10.1210 /$ jc.2015-1520

14 Farnier M, Jones P, Severance R, Averna M, SteinhagenThiessen E, Colhoun HM, Du Y, Hanotin C and Donahue S: Efficacy and safety of adding alirocumab to rosuvastatin versus adding ezetimibe or doubling the rosuvastatin dose in high cardiovascular-risk patients: The ODYSSEY OPTIONS II randomized trial. Atherosclerosis 244(1): 138-146, 2016. PMID: 26638010. DOI: 10.1016/j.atherosclerosis.2015.11.010

15 Cannon CP, Cariou B, Blom D, McKenney JM, Lorenzato C, Pordy R, Chaudhari U, Colhoun HM and ODYSSEY COMBO II Investigators: Efficacy and safety of alirocumab in high cardiovascular risk patients with inadequately controlled hypercholesterolaemia on maximally tolerated doses of statins: The ODYSSEY COMBO II randomized controlled trial. Eur Heart J 36(19): 1186-1194, 2015. PMID: 25687353. DOI: 10.1093/eurheartj/ehv028

16 Raal FJ, Stein EA, Dufour R, Turner T, Civeira F, Burgess L, Langslet G, Scott R, Olsson AG, Sullivan D, Hovingh GK, Cariou B, Gouni-Berthold I, Somaratne R, Bridges I, Scott R, Wasserman SM and Gaudet D: PCSK9 inhibition with evolocumab (AMG 145) in heterozygous familial hypercholesterolaemia (RUTHERFORD-2): A randomised, double-blind, placebo-controlled trial. Lancet 385(9965): 331-340, 2015. PMID: 25282519. DOI: 10.1016/S01406736(14)61399-4

17 Giugliano RP, Mach F, Zavitz K, Kurtz C, Im K, Kanevsky E, Schneider J, Wang H, Keech A, Pedersen TR, Sabatine MS, Sever PS, Robinson JG, Honarpour N, Wasserman SM, Ott BR and EBBINGHAUS Investigators: Cognitive function in a randomized trial of evolocumab. N Engl J Med 377(7): 633-643, 2017. PMID: 28813214. DOI: 10.1056/NEJMoa1701131
18 Giugliano RP, Wiviott SD, Blazing MA, De Ferrari GM, Park JG, Murphy SA, White JA, Tershakovec AM, Cannon CP and Braunwald E: Long-term safety and efficacy of achieving very low levels of low-density lipoprotein cholesterol: A prespecified analysis of the IMPROVE-IT trial. JAMA Cardiol 2(5): 547-555, 2017. PMID: 28291866. DOI: 10.1001/jamacardio.2017.0083

19 Luo J, Yang H and Song BL: Mechanisms and regulation of cholesterol homeostasis. Nat Rev Mol Cell Biol 21(4): 225-245, 2020. PMID: 31848472. DOI: 10.1038/s41580-019-0190-7

20 Faselis C, Imprialos K, Grassos H, Pittaras A, Kallistratos M and Manolis A: Is very low LDL-C harmful? Curr Pharm Des 24(31): 3658-3664, 2018. PMID: 30295187. DOI: 10.2174/ 1381612824666181008110643

21 Robinson JG, Rosenson RS, Farnier M, Chaudhari U, Sasiela WJ, Merlet L, Miller K and Kastelein JJP: Safety of very low low-density lipoprotein cholesterol levels with alirocumab: Pooled data from randomized trials. J Am Coll Cardiol 69(5): 471-482, 2017. PMID: 28153102. DOI: 10.1016/j.jacc. 2016.11.037

22 Toth PP, Descamps O, Genest J, Sattar N, Preiss D, Dent R, Djedjos C, Wu Y, Geller M, Uhart M, Somaratne R and Wasserman SM: PROFICIO: Pooled safety analysis of evolocumab in over 6000 patients from double-blind and open-label extension studies clinical perspective. Circulation 135(19): 1819-1831, 2017. PMID: 28249876. DOI: 10.1161/CIRCULATIONAHA.116. 025233

23 Lipinski MJ, Benedetto U, Escarcega RO, Biondi-Zoccai G, Lhermusier T, Baker NC, Torguson R, Brewer HB Jr and Waksman R: The impact of pro-protein convertase subtilisinkexin type 9 serine protease inhibitors on lipid levels and outcomes in patients with primary hypercholesterolaemia: A network meta-analysis. Eur Heart J 37(6): 536-545, 2016. PMID: 26578202. DOI: 10.1093/eurheartj/ehv563

24 Kołodziejczak M and Navarese EP: Role of PCSK9 antibodies in cardiovascular disease: Critical considerations of mortality and neurocognitive findings from the current literature. Atherosclerosis 247(4): 189-192, 2016. PMID: 26926598. DOI: 10.1016/j.atherosclerosis.2016.02.011

Received May 14, 2020

Revised July 20, 2020

Accepted July 24, 2020 